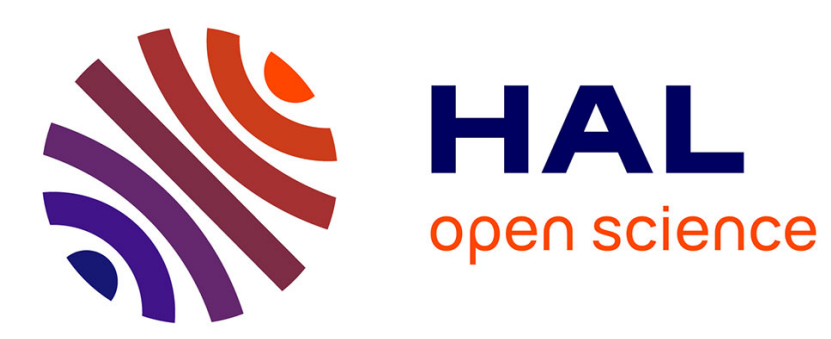

\title{
Fitness Landscape Analysis for Scalable Multicast RRM Problem in Cellular Network
}

\author{
Qing Xu, Frédéric Lassabe, Hakim Mabed, Alexandre Caminada
}

\section{To cite this version:}

Qing Xu, Frédéric Lassabe, Hakim Mabed, Alexandre Caminada. Fitness Landscape Analysis for Scalable Multicast RRM Problem in Cellular Network. INOC 2013, Int. Network Optimization Conf., Jan 2013, Spain. pp.407 - 414. hal-00956237

\section{HAL Id: hal-00956237 \\ https://hal.science/hal-00956237}

Submitted on 6 Mar 2014

HAL is a multi-disciplinary open access archive for the deposit and dissemination of scientific research documents, whether they are published or not. The documents may come from teaching and research institutions in France or abroad, or from public or private research centers.
L'archive ouverte pluridisciplinaire HAL, est destinée au dépôt et à la diffusion de documents scientifiques de niveau recherche, publiés ou non, émanant des établissements d'enseignement et de recherche français ou étrangers, des laboratoires publics ou privés. 


\title{
Fitness Landscape Analysis for Scalable Multicast RRM Problem in Cellular Network
}

\author{
Qing $\mathrm{Xu}{ }^{\mathrm{a}, 1}$, Frédéric Lassabe ${ }^{\mathrm{a}, 1}$, Hakim Mabed ${ }^{\mathrm{b}, 2}$ and \\ Alexandre Caminada ${ }^{\text {a,1 }}$ \\ a IRTES-SeT, Université de Technologie de Belfort-Montbéliard, \\ 13 Rue Thierry Mieg 90010 Belfort, France \\ b FEMTO-ST, Université de Franche-Comté, \\ 1 Rue Claude Goudimel 25030 Montbéliard, France
}

\begin{abstract}
This paper aims to solve the Radio Resource Management (RRM) problem for Multimedia Broadcast Multicast Service (MBMS) system in cellular network. We develop a flexible model to perform dynamic radio resource allocation for MBMS service by using metaheuristic approach. We conduct fitness landscape analysis to study the characteristics of the proposed model, which helps us to select appropriate search strategy. Simulation results show that the proposed algorithm provides better performance than existing algorithms.
\end{abstract}

Keywords: fitness landscape, metaheuristic approach, multimedia multicast, radio resource management.

$\overline{1 \text { Email: }}$ qing.xu, frederic.lassabe, alexandre.caminada@utbm.fr

2 Email: hakim.mabed@pu-pm.univ-fcomte.fr 


\section{Introduction}

The MBMS service over UMTS could be carried by PTM or PTP mode in a base station. A cell is defined to be the geographic coverage of a UMTS base station. In PTM mode, service is transmitted by a forward access channel $(\mathrm{FACH})$ covering the whole cell. Each FACH needs one channel code serving large amount of users, but may waste power when users are close to the base station [4]. The PTP mode uses dedicated channel (DCH) or shared channel (HS-DSCH). Each DCH needs one channel code serving one dedicated user. A HS-DSCH occupies up to 15 channel codes. PTP mode is more efficient than PTM but the served user number is limited. In UMTS where the radio resources (transmit power and channelization codes) are limited, the transmission mode selection is crucial. To improve the efficiency, MBMS Power Counting (MPC) [2] proposes to switch from PTP to PTM when pre-estimated power exceeds an operator-defined threshold, and vice versa. MPC is simple to implement but has very limited flexibility since it does not consider multimedia scalability. Dual Transmission Mode (DTM) allows the mixture of PTP and PTM mode for a same service [5]. However, simulation shows that it is only beneficial for up to 5 users [10]. Scalable FACH Transmission (S-FACH) [6] transmits scalable encoded service through PTM mode. Services are divided into single layer (SL) and multiple layer (ML) transmission schemes, the latter is divided into several flows. The basic flow is transmitted with lower bit rate while the advanced flows with smaller but fixed geographical coverages. However, S-FACH is still based on the fixed coverage so that the resource allocation can not be optimal.

Consider the quite dynamic property of the RRM problem, all mentioned existing approaches provide only limited flexibility. Therefore, the power and date rate allocation can not be optimal. To address this problem, we propose a Flexible Radio Resource Management Model (F2R2M) by using metaheuristic approach. This model combines two objectives: to satisfy the QoS requirement of multicast service and to minimize the power consumption while avoiding saturation of channel code occupation. To understand the behavior of the proposed model, we conduct fitness landscape analysis on the model under five different scenarios. Based on the analysis results, we evaluate two search operators, and compare the performance with existing approaches.

This paper is structured as follows. The proposed model is formulated in section 2. The fitness landscape analysis and the simulation results are given in section 3. The conclusion is drawn in section 4 . 


\section{Model Description}

We focus on multicast transmission within one cell $c$, which is the geographic coverage of a base station. The input variables are defined as follows: $T(c)=$ $\left\{t_{1}, \ldots t_{N t}\right\}$ is a set of users located in $c$, and their instantaneous geometry coordinates $C(c)=\left\{\left(x_{1}, y_{1}\right), \ldots,\left(x_{N t}, y_{N t}\right)\right\}$. A set of services $S(c)=\left\{s_{1}, \ldots, s_{N s}\right\}$ and their the flows $F\left(s_{i}\right)=\left\{f_{s_{i}, 0}\right\}$ or $\left\{f_{s_{i}, 1}, f_{s_{i}, 2}\left[f_{s_{i}, 3}\right]\right\}$ are transmitted in $c$. $f_{s_{i}, 0}$ indicates $s_{i}$ is SL transmission scheme. $f_{s_{i}, j}(j>0)$ is the sublayers of ML scheme service, $f_{s_{i}, 1}$ is the basic flow and $f_{s_{i}, j}(j>1)$ is the advanced flow which is optional for quality improvement. Each user group subscribing to the same service is called a multicast group: $\operatorname{Dist}\left(s_{i}\right)=\left\{t_{n}, t_{m}, \ldots\right\}$.

F2R2M supports the co-existing of PTM and PTP modes for each flow. It partitions Dist $(s)$ into four disjointed sets: users served through a FACH $\mathrm{UE}_{\mathrm{fach}}\left(\mathrm{f}_{\mathrm{s}, \mathrm{j}}\right)$; users transfered through $\mathrm{DCHs} \mathrm{UE}_{\mathrm{dch}}\left(\mathrm{f}_{\mathrm{s}, \mathrm{j}}\right)$; users sharing HS$\mathrm{DSCH} \mathrm{UE}_{\mathrm{hs}}\left(\mathrm{f}_{\mathrm{s}, \mathrm{j}}\right)$ and non-served users $\mathrm{UE}_{\text {noch }}\left(\mathrm{f}_{\mathrm{s}, \mathrm{j}}\right)$. The decision of user set follows two principles: 1) to guarantee the service coverage, all users in multicast group should be selected to receive $f_{s_{i}, 0}$ or $\left.f_{s_{i}, 1}, 2\right)$ in ML transmission, the advanced flow is sent only when a lower flow has been received by users. The partition of user depends on channel characteristics: 1) FACH can be listened by all users within its coverage, hence $\mathrm{UE}_{\mathrm{fach}}\left(\mathrm{f}_{\mathrm{s}, \mathrm{j}}\right)$ includes the nearest group of users in Dist(s), with the distance from the base station under a threshold $d_{t h r} . d_{t h r}$ is dynamically determined during search procedure. 2) the users in $\operatorname{Dist}\left(f_{s, j}\right)$ farther than $d_{t h r}$, are assigned to $\mathrm{UE}_{\mathrm{dch}}\left(\mathrm{f}_{\mathrm{s}, \mathrm{j}}\right)$ or $\mathrm{UE}_{\mathrm{hs}}\left(\mathrm{f}_{\mathrm{s}, \mathrm{j}}\right)$. Consequently, according to the user partition and flow bandwidth, available channel code(s) is associated with a nonempty user set. When no channel code is available for a given user, this user is switched to $\mathrm{UE}_{\text {noch }}\left(\mathrm{f}_{\mathrm{s}, \mathrm{j}}\right)$.

A solution is defined as variable $x$, which is a matrix with $N t$ (number of flows) rows and $N f$ (number of users) columns:

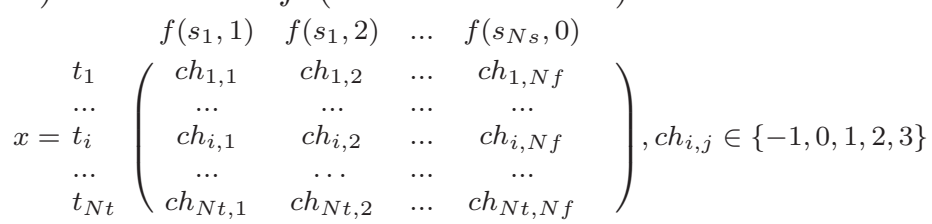

$x(i, j)$ indicates the channel allocation of user $t_{i}$ for flow $f_{j}$. Values 0,1 , 2, 3 represent four possible user allocations: $\mathrm{UE}_{\text {noch,fach,dch,hs }}\left(\mathrm{f}_{\mathrm{s}, \mathrm{j}}\right) .-1$ means the user does not belong to the multicast group. Hamming distance is used to measure the distance of two feasible solutions, which will be used in the calculation of indicators for fitness landscape analysis.

The power consumption is implicitly determined once user and channel code allocation are determined. As simulated in [1], $\mathrm{P}_{\mathrm{FACH}}$ can be got through 
a look up table based on the user distribution in $\mathrm{UE}_{\mathrm{fach}}\left(\mathrm{f}_{\mathrm{s}, \mathrm{j}}\right)$. The DCH transmission power for $n$ users [9] is calculated by : $P_{\mathrm{DCH}}=\frac{P_{p}+\sum_{i=1}^{n} L p_{i} \cdot \frac{P_{n}+I_{i}}{\left(E_{b} / N_{o}\right) R_{b, i}}+p}{1-\sum_{i=1}^{n} \frac{p}{\left(E_{b} / N_{o}\right) R_{b, i}}+p}$, where $P_{p}$ is common control channel power, $P_{n}$ is the background noise, $L p_{i}$ is user path loss, $W$ is the bandwidth in UMTS, $R_{b, i}$ is the transmit rate of $i$ th user, $p$ is the orthogonality factor, $I_{i}$ is the intercell interference observed by $i$ th user. The transmit power to guarantee the required HS-DSCH throughput [8] is: $P_{\mathrm{HS}-\mathrm{DSCH}} \geq \mathrm{SINR} \times\left[\mathrm{p}-\mathrm{G}^{-1}\right] \frac{\mathrm{P}_{\mathrm{own}}}{16}$, in which $P_{\mathrm{own}}$ is the own cell interference experienced by user, $G$ is the geometry factor defined by $G=\frac{P_{\text {own }}}{P_{\text {other }}+P_{\text {noise }}}$, related with the user position.

A two-dimensional fitness value $F(x)=\{T h(x), P o(x)\}$ is defined to measure the solution quality. $P o(x)$ is the MBMS transmission power: $P o(x)=$ $\sum_{s_{i} \in S(c)} \sum_{f_{j} \in F\left(s_{i}\right)} \sum_{c h_{l}} P_{f_{j}, c h_{l}}$. Th(x) is the throughput loss in cell: $T h(x)=$ $\sum_{s_{i} \in S(c)} \sum_{f_{j} \in F\left(s_{i}\right)} \sum_{t_{u} \in \operatorname{Dist}\left(f_{s_{i}, j}\right)} \max \left[-\Delta_{j, u}, 0\right] . \Delta_{j, u}$ is the difference between allocated channel bit rate (determined by its channel code(s) [3]) and flow bandwidth. The optimization target is to first satisfy the throughput requirement within the maximum power budget, then to minimize the transmission power. Therefore, the comparison of a new solution $x^{\prime}$ and current solution $x$ is conducted in lexicographic order: $x^{\prime}$ is evaluated as a better solution when $T h\left(x^{\prime}\right)=T h(x)$ and $P o\left(x^{\prime}\right) \leq P o(x)$, or $T h\left(x^{\prime}\right)<T h(x)$.

We propose two operators to move solution into a neighbor solution.

- Hybrid-moves operator $\delta_{H}$ randomly selects user $t_{k}$ from a non-empty $\mathrm{UE}_{\mathrm{ch}_{\mathrm{o}}}\left(\mathrm{f}_{\mathrm{s}, \mathrm{j}}\right)$, then $t_{k}$ or a block of users including $t_{k}$ is moved from $\mathrm{UE}_{\mathrm{ch}_{\mathrm{o}}}\left(\mathrm{f}_{\mathrm{s}, \mathrm{j}}\right)$ to $\mathrm{UE}_{\mathrm{ch}_{\mathrm{i}}}\left(\mathrm{f}_{\mathrm{s}, \mathrm{j}}\right)$. The moved users depends on the chosen set. For example, if $c h_{i}$ is $\mathrm{FACH}$, the coverage is enlarged to $t_{k}$, all the users nearer than $t_{k}$ can now hear from FACH, thus, no matter what user sets they are currently allocated at, they should stay or be inserted in $\mathrm{UE}_{\mathrm{fach}}\left(\mathrm{f}_{\mathrm{s}, \mathrm{j}}\right)$.

- Insert operator $\delta_{I}$ moves only one user for each operator application. Once it chooses FACH as $c h_{i}$ or $c h_{o}$, then $t_{k}$ is determined: the nearest user in $\mathrm{UE}_{\mathrm{hs}}\left(\mathrm{f}_{\mathrm{s}, \mathrm{j}}\right) \cup \mathrm{UE}_{\mathrm{dch}}\left(\mathrm{f}_{\mathrm{s}, \mathrm{j}}\right) \cup \mathrm{UE}_{\text {noch }}\left(\mathrm{f}_{\mathrm{s}, \mathrm{j}}\right)$, or the farthest user within $\mathrm{UE}_{\mathrm{fach}}\left(\mathrm{f}_{\mathrm{s}, \mathrm{j}}\right)$.

\section{Fitness Landscape of F2R2M}

In Table 1, five test scenarios with different service configurations and user numbers are designed for simulations. For example, in the first scenario $2 s-$ 50-sn, two services $s_{1}$ and $s_{2}$ are transmitted to 30 and 20 users respectively. $s_{1}$ consists of three flows: $f_{1}, f_{2}$ at $32 \mathrm{kbps}$, and $f_{3}$ at $64 \mathrm{kbps} . s_{2}$ consists of only 
one flow $f_{0}$ at $128 \mathrm{kbps}$. The users are assumed to be uniformly distributed around the base station.

Table 1

Simulation scenarios

\begin{tabular}{l|l|l}
\hline Scenarios & Service number, Flow bandwidth & User number per service \\
\hline scen 1: 2s-50u-sn & $2, s_{1}\left(f_{1}, f_{2}: 32 k b p s, f_{3}: 64 k b p s\right) ; s_{2}\left(f_{0}: 128 k b p s\right)$ & 30,20 \\
scen 2: 2s-50u-ss & $2, s_{1}\left(f_{1}, f_{2}: 32 k b p s, f_{3}: 64 k b p s\right) ; s_{2}\left(f_{1}, f_{2}: 64 k b p s\right)$ & 30,20 \\
scen 3: 3s-80u-snn & $3, s_{1}\left(f_{1}, f_{2}: 32 k b p s, f_{3}: 64 k b p s\right) ; s_{2}\left(f_{0}: 128 k b p s\right) ; s_{3}\left(f_{0}: 64 k b p s\right)$ & $30,20,30$ \\
scen 4: 3s-80u-ssn & $3, s_{1}\left(f_{1}, f_{2}: 32 k b p s, f_{3}: 64 k b p s\right) ; s_{2}\left(f_{1}, f_{2}: 64 k b p s\right) ; s_{3}\left(f_{0}: 64 k b p s\right)$ & $30,20,30$ \\
scen 5: 3s-100u-ssn & $3, s_{1}\left(f_{1}, f_{2}: 32 k b p s, f_{3}: 64 k b p s\right) ; s_{2}\left(f_{1}, f_{2}: 64 k b p s\right) ; s_{3}\left(f_{0}: 64 k b p s\right)$ & $30,40,30$ \\
\hline
\end{tabular}

In our model, two fitness landscapes $L_{h y}$ and $L_{i n}$ are defined by $\delta_{H}$ and $\delta_{I}$. We study their characteristics in three aspects: 1) the distribution of feasible solutions within search space; 2) the distribution of fitness space; and 3) the links between distance and fitnesses of solutions. Two populations of solution $S_{i n i}$ and $S_{l o}$ are generated. $S_{i n i}$ is composed of 600 solutions randomly chosen from the initial solution space. $S_{l o}$ is the population of local optima found by applying hill climbing (HC) [7] to $S_{\text {ini }}$. Starting from a random solution in $S_{\text {ini }}, \mathrm{HC}$ evaluates all its feasible neighbors and replaces it by the neighbor that has the best fitness. HC stops at $S_{l o}$ when no neighbor is better than current solution.

\subsection{Analysis of Search Space}

To study the distribution of feasible solutions in search space, two types of distance are calculated: $d_{i n i}$ and $d_{l o}$ are the distances among any two solutions in $S_{i n i}$ and $S_{l o}$. Table 2 presents the minimum, the maximum and the quartiles

Table 2

Analysis of Search Space: distance between solutions of landscapes

\begin{tabular}{|c|c|c|c|c|c|c|c|c|c|c|c|c|}
\hline & \multicolumn{3}{|c|}{$L_{i n}: d_{i n i}$ in $S_{i n i, i n}$} & \multicolumn{3}{|c|}{$L_{i n}: d_{l o}$ in $S_{l o, i n}$} & \multicolumn{3}{|c|}{$L_{h y}: d_{i n i}$ in $S_{i n i, h y}$} & \multicolumn{3}{|c|}{$L_{h y}: d_{l o}$ in $S_{l o, h y}$} \\
\hline & Min & Quartiles & Max & Min & Quartiles & Max & Min & Quartiles & $\operatorname{Max}$ & Min & Quartiles & $\operatorname{Max}$ \\
\hline scen 1 & 15 & $62_{54,72}$ & 109 & 17 & $62_{54,71}$ & 108 & 19 & $62_{53,71}$ & 108 & 0 & $34_{23,45}$ & 109 \\
\hline scen 2 & 20 & $64_{55,74}$ & 114 & 20 & $65_{55,75}$ & 117 & 23 & $64_{54,73}$ & 121 & 0 & $33_{22,46}$ & 127 \\
\hline scen 3 & 20 & 6455,73 & 116 & 20 & $64_{55,74}$ & 116 & 21 & $65_{54,73}$ & 115 & 0 & $43_{30,57}$ & 138 \\
\hline scen 4 & 26 & $66_{56,76}$ & 148 & 20 & $66_{56,76}$ & 148 & 20 & $64_{55,73}$ & 116 & 0 & $35_{24,49}$ & 156 \\
\hline scen 5 & 21 & $64_{55,73}$ & 130 & 19 & $65_{55,76}$ & 180 & 21 & $64_{55,73}$ & 129 & 0 & $38_{23,56}$ & 194 \\
\hline
\end{tabular}

(median, Q1 and Q3) of these distances. Firstly, for both operators, the distance statistics in the initial solution space $\left(d_{i n i}\right.$ in $S_{i n i, i n}$ and $\left.S_{i n i, h y}\right)$ are homogeneous, that is simply because they are randomly selected in the initial 
solution space. Secondly, when looking at the the distance statistics in the local optima space $\left(d_{l o}\right.$ in $S_{l o, i n}$ and $\left.S_{l o, h y}\right), \delta_{H}$ generates much converged space.

\subsection{Analysis of Fitness Space}

Figure 1 illustrates the fitness value distribution of $S_{i n i}$ and $S_{l o}$ for the fifth scenario 3s-100u-ssn. The fitness values of $S_{i n i}$ are well diversified for both operators. Comparing the first and third subfigures, the quality of $S_{l o, h y}$ is
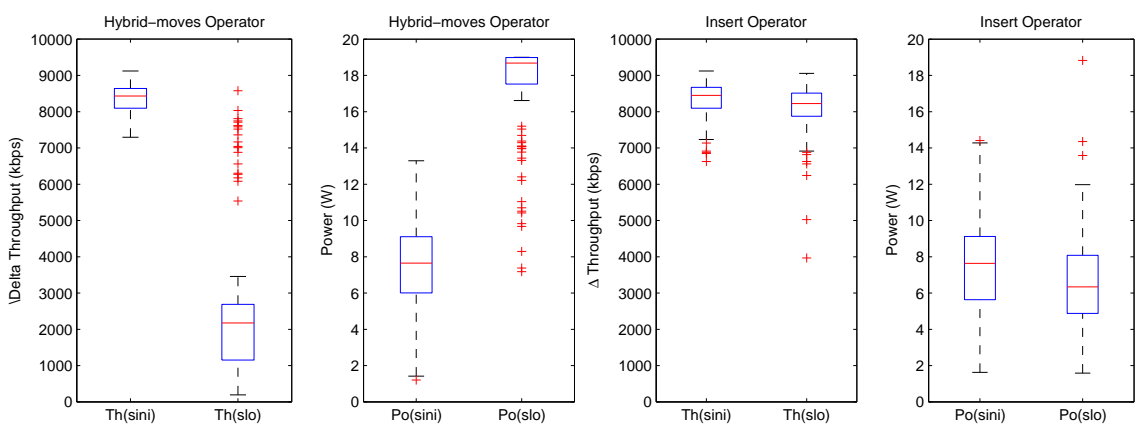

Fig. 1. Analysis of Fitness Space: Comparison of two fitness space (3s-100u-ssn)

better than that of $S_{l o, i n}\left(T h_{h y}(l o)<T h_{i n}(l o)\right)$. The same situation for the other scenarios is confirmed in Table 3 , in which we can see $\delta_{H}$ finds solutions with smaller $T h(x)$, hence better solutions than $\delta_{I}$. This can be explained by the fact that, unlike $\delta_{I}, \delta_{H}$ moves a block of elements which explores larger search space, so that it can easily escape from the local optimal.

Table 3

Fitness space analysis: fitness values of populations of local optima

\begin{tabular}{|c|c|c|c|c|c|c|c|c|c|}
\hline \multirow[t]{2}{*}{ Scenarios } & \multirow{2}{*}{ Fitness } & \multicolumn{4}{|c|}{$L_{h y}: S_{l o, h y}$} & \multicolumn{4}{|c|}{$L_{i n}: S_{l o, i n}$} \\
\hline & & Min & $\operatorname{Med}_{\mathrm{Q1}, \mathrm{Q3}}$ & $\operatorname{Max}$ & Mean & Min & $\operatorname{Med}_{\mathrm{Q} 1, \mathrm{Q} 3}$ & $\operatorname{Max}$ & Mean \\
\hline \multirow{2}{*}{$2 \mathrm{~s}-50 \mathrm{u}-\mathrm{sn}$} & $\operatorname{Th}(\mathrm{x}) \%$ & 0 & $0_{0,0}$ & 58 & 1.7 & 4.5 & $57.5_{52.25,62}$ & 69 & 56.21 \\
\hline & $\operatorname{Po}(x) w$ & 7.08 & $14.89_{12.95,18.18}$ & 19.0 & 15.28 & 1.5 & $6.53_{5.03,8.33}$ & 18.54 & 6.44 \\
\hline \multirow{2}{*}{$2 \mathrm{~s}-50 \mathrm{u}-\mathrm{ss}$} & $\operatorname{Th}(x) \%$ & 0 & $0_{0,0}$ & 62.5 & 0.94 & 0 & $58.75_{54,62}$ & 69.5 & 10.74 \\
\hline & $\operatorname{Po}(x) w$ & 5.81 & $14.63_{14.36,15.02}$ & 16.58 & 14.57 & 1.69 & $6.24_{4.69,7.93}$ & 16.3 & 6.44 \\
\hline \multirow{2}{*}{$3 \mathrm{~s}-80 u-\operatorname{snn}$} & $\operatorname{Th}(\mathrm{x}) \%$ & 0 & $4_{0,15}$ & 91.5 & 9.43 & 37 & $87.5_{82,92}$ & 99 & 86.61 \\
\hline & $\operatorname{Po}(x) w$ & 5.543 & $17.29_{15.92,18.79}$ & 18.99 & 17.2 & 2.10 & $6.56_{4.96,8.21}$ & 18.96 & 6.74 \\
\hline \multirow{2}{*}{$3 s-80 u-s s n$} & $\operatorname{Th}(x) \%$ & 0 & $1_{0,2}$ & 93.5 & 3.8 & 20.5 & $88_{83.5,91.5}$ & 99 & 86.34 \\
\hline & $\operatorname{Po}(x) w$ & 6.38 & $17.57_{17.22,17.63}$ & 19.0 & 17.2 & 2.1 & $6.56_{4.96,8.21}$ & 18.96 & 6.75 \\
\hline
\end{tabular}




\subsection{Analysis of Links between Distance and Fitness}

In F2R2M, step length is the number of implemented operator applications by using hill climbing method. The average step length in $L_{h y}$ for five scenarios are: $26,27,43,68,100$. While in $L_{i n}$, they are: $10,10,9,11,13$. In general, $\delta_{I}$ moves shorter distance than $\delta_{H}$, which makes the search procedure walks nearby the initial solution. This explains that the search space of $S_{l o, \text { in }}$ does not concentrate the $S_{i n i, i n}$ (Table 2). Therefore, $L_{i n}$ is "shallower" than $L_{h y}$.

\subsection{Results Comparison with Conventional Algorithms}

The solutions of competing approaches and the best found solutions based on F2R2M are shown in Table 4. Solutions are represented in two-dimensional: $T h(x)$ in percentage and $P o(x)$ in watts. The maximum power budget is set as $19 \mathrm{w}$ hence solutions with $T h(x)<19$ are feasible and emphasized in boldface. Both operators in F2R2M can always find good enough solutions: less throughput loss with less power consumption than existing approaches. The mean fitness values of found solution with F2R2M-in for scenarios 2 to 5

Table 4

Results comparison with existing approaches

\begin{tabular}{l|l|l|l|l|l}
\hline Scenarios & $\begin{array}{l}\text { MPC } \\
\{T h(x), P o(x)\}\end{array}$ & $\begin{array}{l}\text { DTM } \\
\{T h(x), P o(x)\}\end{array}$ & $\begin{array}{l}\text { S-FACH } \\
\{T h(x), P o(x)\}\end{array}$ & $\begin{array}{l}\text { F2R2M-in } \\
\{T h(x), P o(x)\}\end{array}$ & $\begin{array}{l}\text { F2R2M-hy } \\
\{T h(x), P o(x)\}\end{array}$ \\
\hline 2s-50u-sn & $0 \%, 27.2$ & $0 \%, 30.5$ & $\mathbf{6 5 \% \mathbf { 1 0 . 2 }}$ & $\mathbf{4 . 5 \% , \mathbf { 1 8 . 5 }}$ & $\underline{\mathbf{0 \%} \mathbf{1 0 . 2}}$ \\
\hline 2s-50u-ss & $0 \%, 27.2$ & $0 \%, 30.5$ & $\mathbf{4 7 \%} \mathbf{1 5 . 4}$ & $\mathbf{0 \% , 1 5 . 5 8}$ & $\underline{\mathbf{0 \%} \mathbf{1 3 . 1}}$ \\
\hline 3s-80u-snn & $0 \%, 32.5$ & $0 \%, 37.7$ & $25.4 \% 27.0$ & $\mathbf{2 5 . 4 \% , \mathbf { 1 6 . 9 }}$ & $\underline{\mathbf{0 \%} \mathbf{1 5 . 0}}$ \\
\hline 3s-80u-ssn & $0 \%, 32.5$ & $0 \%, 37.7$ & $36.2 \% 22.6$ & $\mathbf{1 5 . 4 \% , \mathbf { 1 6 . 5 }}$ & $\underline{\mathbf{0 \%} \mathbf{1 4 . 4}}$ \\
\hline 3s-100u-ssn & $0 \%, 37.73$ & $0 \%, 37.69$ & $\mathbf{5 1 . 1 8 \% , \mathbf { 1 5 . 9 }}$ & $\mathbf{1 5 . 9 \% , \mathbf { 1 8 . 1 2 }}$ & $\underline{\mathbf{1 . 7 6 \% , \mathbf { 1 7 . 5 }}}$ \\
\hline
\end{tabular}

are from $(56.2 \%, 6.4 \mathrm{w})$ to $(74.4 \%, 6.65 \mathrm{w})$ with standard deviation from $(8.12$, $2.45)$ to $(7.13,2.55)$. While for F2R2M-hy, the mean fitness values are from $(1.7 \%, 15.3 \mathrm{w})$ to $(7.29 \%, 18.12 \mathrm{w})$ with standard deviation from $(8.2,2.56)$ to $(11.93,1.4) . \delta_{H}$ performs better than $\delta_{I}$, which proves the discussion in section 3 that $\delta_{H}$ has capacity of "jump" from bad solutions, while $\delta_{I}$ can only stay in basins. Besides, the average consuming time of one $\mathrm{HC}$ trial is calculated, $\delta_{I}$ and $\delta_{H}$ needs $0.06 \mathrm{~s}$ and $0.16 \mathrm{~s}$ for $2 s-50 u-s n, 0.77 \mathrm{~s}$ and $0.31 \mathrm{~s}$ for 3s-100u-ssn. Both operators can find good enough solution with acceptable time. $\delta_{H}$ can move more steps hence farther than $\delta_{I}$, therefore the hybridmoves operator costs double time than insert operator. 


\section{Conclusion and Perspective}

In order to solve the RRM problem in MBMS system, we propose a novel model named Flexible Radio Resource Management Model (F2R2M) using metaheuristic approach. In our model, two objectives are took into account to satisfy the QoS request and minimize the power consumption. Based on fitness landscape analysis, a study is investigated on the performance of the two proposed neighborhood functions. Simulation results show that our approaches can achieve significant gains comparing with the other existing approaches. In the future work, we are interested in applying the other local search algorithms such as Simulated Annealing and Tabu Search to improve the search efficiency.

\section{References}

[1] 3GPP Standards: TR 25.803 S-CCPCH performance for MBMS, Sep 2005.

[2] 3GPP Standards: TR 25.922 radio resource management strategies, Mar 2007.

[3] 3GPP Standards: TS 25.213 spreading and modulation (FDD), December 2009.

[4] A. Alexiou, C. Bouras, and E. Rekkas. A power control scheme for efficient radio bearer selection in MBMS. In World of Wireless, Mobile and Multimedia Networks, 200\%. WoWMoM 200\%. IEEE International Symposium on, pages $1-8$, June.

[5] C. Christophorou, A. Pitsillides, and T. Lundborg. Enhanced radio resource management algorithms for efficient MBMS service provision in UTRAN. Computer Networks, 55(3), 2011.

[6] A. M. C. Correia, J. C. M. Silva, N. M. B. Souto, L. A. C. Silva, A. B. Boal, and A. B. Soares. Multi-resolution broadcast/multicast systems for MBMS. Broadcasting, IEEE Transactions on, 53(1):224-234, Mar. 2007.

[7] F. W. Glover and G. A. Kochenberger, editors. Handbook of Metaheuristics. Springer, 1 edition, Jan. 2003.

[8] A. T. Harri Holma. HSDPA/HSUPA for UMTS: High Speed Radio Access for Mobile Communications. John Wiley \& Sons, 2006.

[9] J. P. Romero, O. Sallent, R. Agusti, and M. A. Diaz-Guerra. Radio Resource Management Strategies in UMTS. Wiley, 1 edition, Aug. 2005.

[10] L. Technologies. 3GPP TSG RAN WG128 R1-02-1240 power usage for mixed FACH and DCH for MBMS, Oct 2002. 